FERMILAB-TM-1983

\title{
Problems in the Design of a Proton Driver for a $\mu-\mu$ Collider
}

\author{
F.E. Mills
}

Fermi National Accelerator Laboratory

P.O. Box 500, Batavia, Illinais 60510

September 1996 


\section{Disclaimer}

This report was prepared as an account of work sponsored by an agency of the United States Government. Neither the United States Government nor any agency thereof, nor any of their emplovees, makes any. warrant, express or implied, or assumes any legal liability or responsibility for the accuracy: completeness or usefulness of any information, apparatus, product or process disclosed, or represents that its use would not infringe privately owned rights. Reference herein to any specific commercial product, process or service by trade name, trademark, manufacturer or otherwise, does not necessarily constitute or imply its endorsement, recommendation or favoring by the United States Government or any agency thereof. The liews and opinions of authors expressed herein do not necessarily state or reflect those of the United States Government or any agency thereof.

\section{Distribution}

Approved for public release: further dissemination unlimited. 
Problems in the Design of a Proton Driver for a $\mu-\mu$ Collider

F. E. Mills

\section{Introduction}

To produce an adequate supply of muons, the goal is to create a bunch hitting a target with $5 \times 10^{13}$ protons and a rms width of 3 nsec. It seems difficult to create such a proton bunch in equilibrium in a ring. For example if the initial bunch at injection is space charge limited, the tune shift will be reduced by the ratio of $\beta \gamma^{2}$ but increased by the ratio of the bunching factor. For the case developed below. $1 \mathrm{GeV}$ (kinetic energy) injection and $8 \mathrm{GeV}$ extraction in a ring of length $1600 \mathrm{nsec}$, the tune shift at extraction is higher than at injection by a factor of four. However, large transient tune shifts have been observed. [1] We might hope to "rotate" transiently the bunch with a longitudinal lens and allow the beam to drift in a ring to compress into a short bunch. provided we do not expect the beam to drift too long.

In the Fermilab Antiproton Debuncher, a 4\% momentum spread is compressed into $0.25 \%$, a substantial ratio of 16 . The present case, however, requires a factor of 133 increase in momentum spread. It does not seem credible that the nonlinearities inherent in the RF wave form will allow such a large ratio. Alternatively, a single pass device. for example in the form similar to an induction linac. might be built at sub harmonic frequency (with longer pulse length) to achieve the required linearity in the field. Further, harmonic cavities can be used to improve linearity. The amount of acceleration and deceleration is dependent on the momentum spread in the proton beam from the driver. as is the momentum acceptance of the buncher ring.

It is interesting to speculate on what the performance would be with present FNAL Booster parameters. At low intensity ( $<10^{10} /$ bunch), the final bunch area is $.02 \mathrm{eVsec}$ [2], while at $2.5 \times 10^{10} /$ bunch it is $.06 \mathrm{eVsec}$. It is not clear that the area increase is due to microwave instability. Debunching until the bunches touch $(\pi / 2$ dilution) gives a proton beam of $1600 \mathrm{nsec}$ length and $1.7 \mathrm{MeV}$ width. To achieve a $12 \mathrm{nsec}$ full width. the energy width must be increased to $225 \mathrm{MeV}$ or $2.5 \%$ in momentum. This might be accomplished with an induction linac device with about $200-250 \mathrm{MeV}$ maximum gain, then drifting in a modest ring. At the higher bunch areas. the linac becomes a bit long. and the ring acceptance becomes uncomfortably large. Accordingly we need to study those things that place limitations on momentum spread and proton number in the driver. Any scheme will be limited by these considerations. 


\section{Accelerator Physics Issues}

\section{A. Space Charge Tune Shift}

The Laslett space charge tuneshift is [3]

$$
\Delta v=\frac{N_{r_{p}} F}{2 \beta^{2} \gamma^{3} \varepsilon B}
$$

where $F=1+$ image terms, $r_{p}=1.53 \times 10^{-18} \mathrm{~m}, \varepsilon$ is the $100 \%$ geometric phase space area, and $B$ is the ratio of the mean and peak currents. For a uniform elliptical beam of semimajor axes $(a>b)$, the factor of 2 in the denominator becomes $1+a / b$. For injection at $1 \mathrm{GeV}, \mathrm{N}=10^{14}, \mathrm{~B}=.4$ and $\varepsilon=120 \pi \mu \mathrm{m}$, the tuneshift is $\Delta v \approx 0.075$, a resonably low value.

At $8 \mathrm{Gev}$ (kinetic energy) the emittance is reduced by the ratio of $\beta \gamma$, so the tune shift is reduced by a factor of $\beta \gamma^{2}$ for the same $B$, or about a factor of $90.2 / 3.74=24.2$. When the beam is to hit a target, the bunching factor $B$ will decrease to about $12 \mathrm{nsec} / 1600$ nsec $=1 / 133$. Then the tune shift at that point will be about $133 \times 0.4 \times 0.075 / 24.2=0.16$. In the second ring, a metal chamber will be acceptable, so the beam magnetic images don't have time to soak through the chamber, and the electric and magnetic image tune shifts still cancel as 1- $\beta^{2}$.

\section{B. Microwave Instability of Bunched Beam}

The "Keil-Schnell" criterion, ignoring niceties of the dispersion equation yields the instability threshold in a beam,

$$
\frac{Z_{\|}}{n}<\frac{F|\eta| \beta^{2} E / e}{I_{p k}}\left(\frac{\Delta P}{P}\right)^{2}
$$

where $\mathrm{n}$ is the mode number, $\mathrm{F} \approx 1$ is the form factor, $\Delta \mathrm{p}$ is the full momentum spread, $\mathrm{\eta}$ is the phase slip factor, $E$ is the total energy and $I_{p k}$ is the peak current. This threshold is apparently exceeded by a factor of ten in coasting beam, and a factor of three in bunched beam in ISIS at the Rutherford Laboratory [4]. There is some disagreement about the reason for this.

For a round beam of radius a in a perfectly conducting straight pipe of radius $b$,

$$
\frac{Z_{\|}}{n}=\frac{-j Z_{0} g_{0}}{2 \beta \gamma^{2}}
$$

where $\mathrm{g}_{0}=1+2 \ln \frac{\mathrm{b}}{\mathrm{a}}, \mathrm{Z}_{0}=377 \Omega$. If the wall is resistive, there is an additional impedance

$$
\begin{aligned}
& \frac{Z_{\| \text {lwall }}}{n}=\frac{(1+j) \rho R \alpha}{\delta b n} \\
& \delta=\sqrt{\frac{2 \rho}{\mu \omega}}, \\
& \alpha=\frac{1+\exp \left(\frac{-2(1-j) d}{\delta}\right)}{1-\exp \left(\frac{-2(1-j) d}{\delta}\right)},
\end{aligned}
$$


$\mathrm{d}$ is the wall thickness, $\rho$ is the resistivity, $\mu$ is the magnetic permeability and $\omega$ is the mode frequency.

The above impedance is due to electric fields on the axis due to free electric charges as well as changing magnetic flux linking the beam. The term inverse in $\gamma^{2}$ is the relativistic cancellation between them typical in non-curved geometry. Several things can change this cancellation, for example curvature, "inductive walls" in which the electric and magnetic "walls" occur at different radii, lumped inductors through which the return or wall currents flow, or a helical wall through which the return current flows, creating a magnetic field in the direction of the beam. All these possibilities change the relative amount of electric and magnetic energy stored in the neighborhood of the beam.

If the magnetic wall is at radius $d=b+t$ instead of $b$, and the magnetic permeability of the region $b<r<d$ is $\mu$, there is an added term

$$
\frac{\Delta Z_{\|}}{n}=2 j \beta Z_{0} \mu \ln \frac{d}{b} \text {. }
$$

This term will cancel that in Eqn. (3) and the total impedance will be zero if

$$
2 \mu \ln \frac{\mathrm{d}}{\mathrm{b}} \approx 2 \mu \frac{\mathrm{t}}{\mathrm{b}}=\frac{\mathrm{g}_{0}}{\beta^{2} \gamma^{2}} \text {. }
$$

For $\gamma=2, \mathrm{~g}_{0}=2, \mathrm{~b}=30 \mathrm{~mm},<\mu \mathrm{t}>\approx 7.5 \mathrm{~mm}$. The problem with this approach is that any candidate magnetic material, such as ferrite, will have high losses at high frequencies giving a large resistive component to the impedance. On the other hand a similar result could be achieved by making most of the chamber in the form of bellows to raise the inductance. Similarly a helical wall at radius b with pitch angle $\alpha$ contributes a term

$$
\frac{\Delta Z_{\|}}{n}=j Z_{0} \frac{\beta}{\alpha^{2}} \text {. }
$$

The value of $\mathrm{g}$ has been reduced to about 1.5 in ISIS by the use of form-fitting conducting rods around the beam to carry the return currents while letting the main magnet flux through to bend the beam. This arrangement is inside a ceramic chamber. At $1 \mathrm{GeV}$, for a beam of $5 \times 10^{13}$ protons in a Booster length machine with a bunching factor of 0.3 , the peak current is $15 \mathrm{~A}$. If $\mathrm{g}=1.5, \mathrm{ml}=0.25$, then from Eqn. (3), $\frac{Z_{\|}}{n}=76 \Omega$, and the Keil-Schnell limit requires $\Delta \mathrm{P} / \mathrm{P}=1.7 \times 10^{-3}$, or $\Delta \mathrm{E}=2.6 \mathrm{MeV}$. The beam phase space area is then $1.4 \mathrm{eVsec}$ which increases to at least $3 \mathrm{eVsec}$ at $8 \mathrm{GeV}$ due to dilution. To bunch this from $1.6 \mu \mathrm{sec}$ to $12 \mathrm{nsec}$ requires increasing the energy spread to $250 \mathrm{MeV}$ with a linear ramp (using for example an induction linac type structure) with about $150 \mathrm{MeV}$ peak energy gain.

Clearly it would be desireable to reduce, and control, the impedance in any device we build, if possible more than that achieved in ISIS. On the other hand, there does not seem to be a better method than reducing " $g$ " as much as possible with the Rutherford rod cage and avoiding transition energy. 
The growth rates above and below transition for the microwave instability are:

$$
\begin{array}{lll}
\frac{1}{\tau}=n \omega_{0}\left[\frac{\eta e \mathrm{I}_{0}}{2 \pi \beta^{2} \mathrm{E}} \frac{X_{\mathrm{n}}}{\mathrm{n}}\right] \frac{1}{2} & \text { for } & \gamma>\gamma_{\mathrm{t}} \\
\frac{1}{\tau}=n \omega_{0}\left[\frac{\eta \mathrm{eI}}{2 \pi \beta^{2} \mathrm{E}} \frac{X_{\mathrm{n}}}{\mathrm{n}}\right] \frac{1}{2} \frac{\mathrm{R}_{\mathrm{n}}}{X_{\mathrm{n}}} & \text { for } & \gamma<\gamma_{t}
\end{array}
$$

It is interesting to compare ISIS (unbunched) with a Mu Driver design $(B=0.3)$ which has $1.6 \mathrm{eV} \sec \left(\frac{\delta \mathrm{P}}{\mathrm{P}}= \pm 10^{-3}\right)$ at injection $(4-5 \mathrm{eV} \mathrm{sec}$ extracted $)$. Both machines operate below transition.

$\begin{array}{lll}\text { Machine } & \text { ISIS } & \text { Mu Driver } \\ \text { Radius }(\mathrm{m}) & 25 & 75 \\ \text { Injection Energy }(\mathrm{MeV}) & 70 & 1000 \\ \text { Revolution Period }(\mu \mathrm{sec}) & 1.43 & 1.8 \\ \mathrm{~N} \text { protons } & 4 \times 10^{13} & 5 \times 10^{13} \\ \frac{\Delta \mathrm{P}}{\mathrm{P}} & 210^{-3} & 2 \times 10^{-3} \\ \beta & 0.366 & 0.875 \\ \beta \gamma^{2} & 0.423 & 3.74 \\ \rho_{\mathrm{e}}(\Omega \mathrm{m}) & 0.47 \times 10^{-6}(\mathrm{SS}) & 1.6 \times 10^{-8}(\mathrm{Cu}) \\ \mathrm{ml} & 0.62 & 0.227 \\ \mathrm{~b}(\mathrm{~cm}) & 7 & 7 \\ \mathrm{~g} & 1.5 & 1.5 \\ \delta_{\mathrm{S}}(\mathrm{mm} \text { at fundamental) } & 0.41(\mathrm{SS} \text { rods }) & 0.09(\mathrm{Cu} \text { wires }) \\ \frac{\mathrm{R}_{\mathrm{n}}}{\mathrm{n}}(\Omega) & \frac{0.4}{\sqrt{\mathrm{n}}} & \frac{0.2}{\sqrt{\mathrm{n}}} \\ \frac{\mathrm{X}_{\mathrm{n}}}{\mathrm{n}}(\Omega) & 667 & 75 \\ \frac{\mathrm{Zn}}{\mathrm{n}} \text { at threshhold }(\Omega) & 76 & 91 \\ \tau(\mathrm{sec}) \text { growth time } & \frac{0.26}{\sqrt{n}} & \frac{0.65}{\sqrt{n}}\end{array}$




\section{Transverse Resistive Wall Instability}

Instability takes place when the real part of the frequency shift $\Delta v$ due to the collective force for mode $n$ exceeds the frequency spread $\delta v$ for that mode:

$$
\begin{aligned}
& \delta v=\ln (n-v)-\xi v \mid \frac{\delta p}{p} f w h m \\
& \Delta v=\frac{\operatorname{ReIZ}}{4 \pi v \beta E}
\end{aligned}
$$

This yeilds the stability limit:

$$
e \mathrm{IZ}_{\perp}<\frac{4 \pi \beta E v}{R}|\eta(n-v)-\xi v| \frac{\delta p}{p} \text { fwhm }
$$

The transverse capacitive impedance of the wall is:

$$
Z_{\perp}=\frac{R Z_{o} g_{\perp}}{\beta^{2} \gamma^{2}}
$$

where $g_{\perp}=\frac{1}{a^{2}}-\frac{1}{b^{2}}$, and the transverse resistive wall impedance is:

$$
\mathrm{Z}_{\perp}=\frac{2 \mathrm{c} \mathrm{Z}_{\text {|wall }}}{\omega \mathrm{b}^{2}}
$$

where $\mathrm{Z} \|$ is given by Eqn. (4).

The growth times are dominated by the kicker magnets, while the thresholds are determined by the space charge impedance. There is a relationship between space charge tune shift and transverse impedance which sets a limit to the ability to stabilize the motion with Landau damping[5]. If the space charge tune shift is at its maximum value, Landau damping will cause this limit to be exceeded. Then a fed back kicker will be needed to stabilize the lower modes.

\section{Head-tail Instability}

This instability is normally treated in proton machines by controlling the chromaticity so that only the $m=0$ mode is unstable. This can be damped with the aforementioned fed back kicker.

\section{E. Cavity Beam Loading}

This subject has been treated in some depth by Jim Griffin[6]. In summary, such systems are stabilized against Robinson Instability by shifting the cavity resonant frequency and the cavity drive current so that the vector sum of the drive current and the beam current 
produces the desired cavity voltage. Below transition the cavity must be tuned above resonance and vice versa. The effective $Q$ of the cavity is controlled by the plate resistance of the driver tube whose operating parameters are chosen appropriately. The power dissipated in the RF system must exceed that given to the beam. For the intensities forseen, this is not difficult to achieve.

Stability of the $\mathrm{h} \pm 1$ mode is determined by the difference of the real cavity impedance at the two freqencies, a quantity made larger or smaller by the shift of cavity resonance frequency. This difference can be controlled by the degree of tuning, as opposed to increased drive current used to achieve the desired voltage.

Multibunch instability due to other ("higher order") cavity modes must be controlled by damping the modes or by feedback systems. Although the Booster cavities are well known in this regard, some further study is needed.

\section{F. Lattice design}

Although there are many detailed requirements for the lattice, such as to optimize injection, obtain large dynamic aperture, accommodate the RF system, locate feedback components etc., the overwhelming need is to avoid crossing transition energy during acceleration. Almost any scheme of impedance and instability control will be frustrated if transition is crossed. Popovic [7] has investigated FODO lattices with many periods which have $\gamma_{t}$ of about 12 and fit in the Booster tunnel. These lattices exhibit the usual problems of short magnets, high phase advances, and will probably exhibit problems with dynamic aperture. A more promising approach by $\mathrm{C}$. Johnstone $[8]$ follows the work of Lee, $\mathrm{Ng}$, and Trbojevic [9] in manipulating an oscillation of the disperion function to lower (or even make negative) the momentum compaction $\alpha=\left(\gamma_{t}\right)^{-2}$. Using this scheme, lattices with $\gamma_{t}=$ j8.5, $\beta_{\max }$ of about $25-35 \mathrm{~m}$, have been found with reasonable (small) dispersion which fit in the Booster tunnel.. This is rather promising and can be pursued to optimize the lattice and incorporate other features needed.

\section{Technical Components}

Some of the results above were based on an assumption that the ISIS-type wire or rod cage was used to minimize the impedance seen by the beam. This seems to have been very successful[10]. There are several choices remaining, however.

\section{A. Vacuum}

The requirement on pressure is not very demanding. ISIS developed a ceramic chamber into which the rod cage was placed. Because the $800 \mathrm{MeV}$ ISIS is installed in a 
tunnel designed for the $6 \mathrm{GeV}$ Nimrod, the increased magnet aperture required by the ceramic chamber did not represent a severe penalty, since the magnets are weak in any case. A satisfactory alternative might be to incorporate the rod cages into a system like the FNAL Booster, i.e. incorporating the magnets into the vacuum with the cage inside. There is another difference from ISIS which needs study. That is, the sagitta in this dipole may be larger than that in ISIS, requiring the cages to have more sharply curved rods.

\section{B. Magnets}

The magnets should not be a serious challenge, but they are larger than those typical of Fermilab. The maximum amplitudes of vertical oscillations occupy $130 \mathrm{~mm}$. To this must be added some allowance for closed orbit error, the rod cage, and the ceramic chamber (if used). A $0.35 \mathrm{~mm}$ thick 1-2\% Silicon steel will be a good choice for magnetic material. The incorporation of "two frequency" excitation should be studied to attempt to reduce the required $\mathrm{RF}$ Voltage.

\section{RF Cavities}

As noted above, the cavity needs a driver tube with a larger anode dissipation than those in use on the Booster. In addition the bore needs to be to be larger to accommodate the larger beam size. Jim Griffin has suggested that the tuners and bias supplies could be the same as those in the present Booster, and that one could imagine reworking the cavities to enlarge the bore. It is not clear that there is enough room in the lattice for the added RF cavities needed to raise the repetition frequency from $15 \mathrm{~Hz}$ to $30 \mathrm{~Hz}$. This issue still needs to be addressed.

\section{Injection}

A tentative design of a $1 \mathrm{GeV} \mathrm{H}^{-}$Linac has been made by $\mathrm{R}$. Noble[11]. It incorporates a $200 \mathrm{MHz}$ DTL followed by an $800 \mathrm{MHz}$ CCL. The output current is 65 $\mathrm{mA}$, while the emittance and energy spread are adequate. A debuncher will be needed to reduce the momentum spread by about a factor of 2.5 .

The injection straight section needs to be somewhat longer than that in the present Booster. Schemes for "painting" the acceptance to minimize space charge effects have been studied extensively at Rutherford and Los Alamos. These were discussed at some length at the Neutron Spallation Source Workshop at Santa Fe in 1993. 


\section{Final Beam Bunching}

Several schemes have been proposed. None has been completely spelled out.

\section{A. Chicane}

In this scheme[12] multiple bunches are accelerated in a ring, compressed and extracted. Kickers cause different bunches to travel on different paths whose lengths differ by the bunch spacing. The paths are recombined by kicker magnets, forming one bunch of the desired length. This bunch then strikes the target. Such a scheme is indicated in Figure 1. The principal problem with this scheme is the length of the beam lines.

\section{B. Assembler Ring with Linear Induction Buncher}

In this scheme there is a proton synchrotron with many (84) bunches. After acceleration, the bunches are adiabatically debunched until neighboring buckets are full, and then extracted. The beam passes through a subharmonic bunching system resembling an induction linac where it receives a linear tilt in energy-time space which causes it to bunch transiently in the assembler ring as suggested in Figure 2.

Here, with parameters above, the final beam area including dilution at capture and debunching prior to extraction may be as large as $5 \mathrm{eVsec}$. Then the final total momentum

spread of a $12 \mathrm{nsec}$ bunch will be about $400 \mathrm{Mev}$, or a $\frac{\Delta \mathrm{P}}{\mathrm{P}}$ of about $5 \%$. If the Assembler Ring has $\eta=.025$, it will take about 1600 revolutions for the beam to assemble before extraction.

The principal problem with this scheme is the bunching system and its cost. In the first place, induction linacs are rather expensive. Second, induction linacs normally run with all the magnetic energy stored in the magnetic core (no air gap). Under these conditions, the energy lost in the core distorts the wave form so that it does not resemble a half sine wave, most of the energy being gone before the end of the pulse. This is acceptable for linacs where PFN's can be devised to obtain an acceptable pulse shape, but not in this application where the voltage is run through zero. The way around this is to put an air gap in the core, raising the stored energy and the " $Q$ ". This certainly raises the cost, and the fringe fields form the air gaps must be configured so as not to deflect the beam. Nevertheless, such a system might be built. 


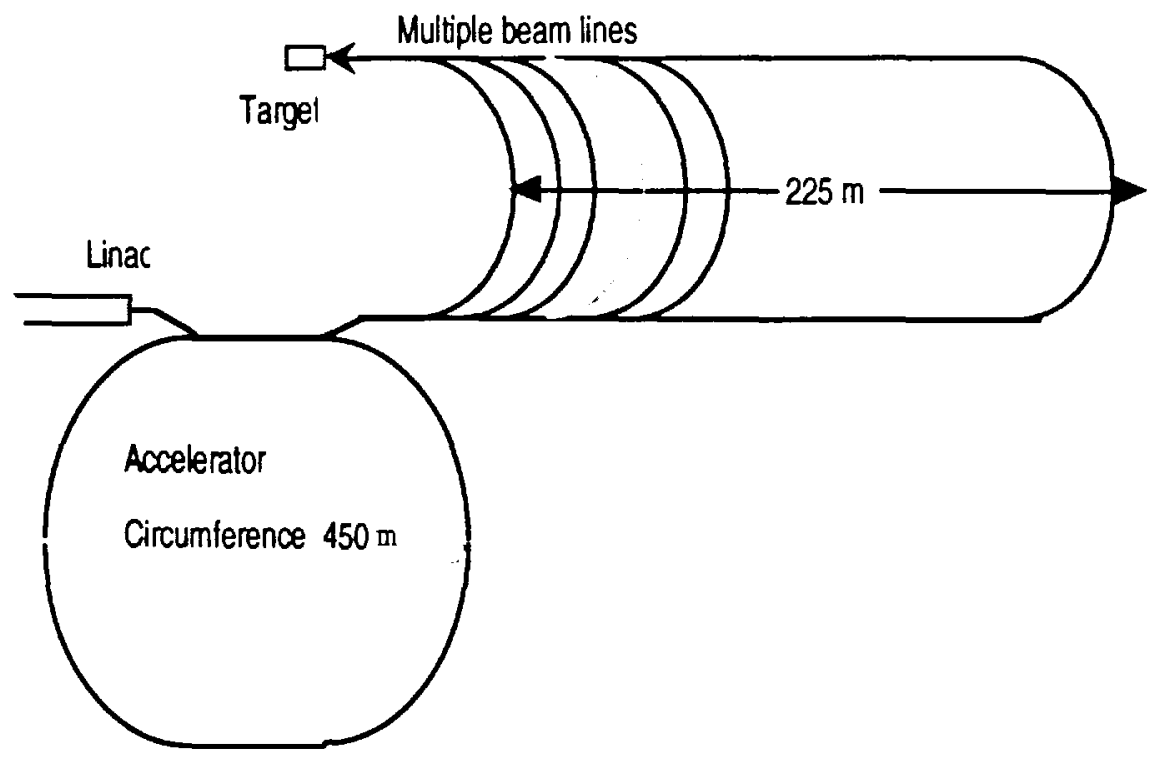

Figure 1. Schematic of Chicane System 


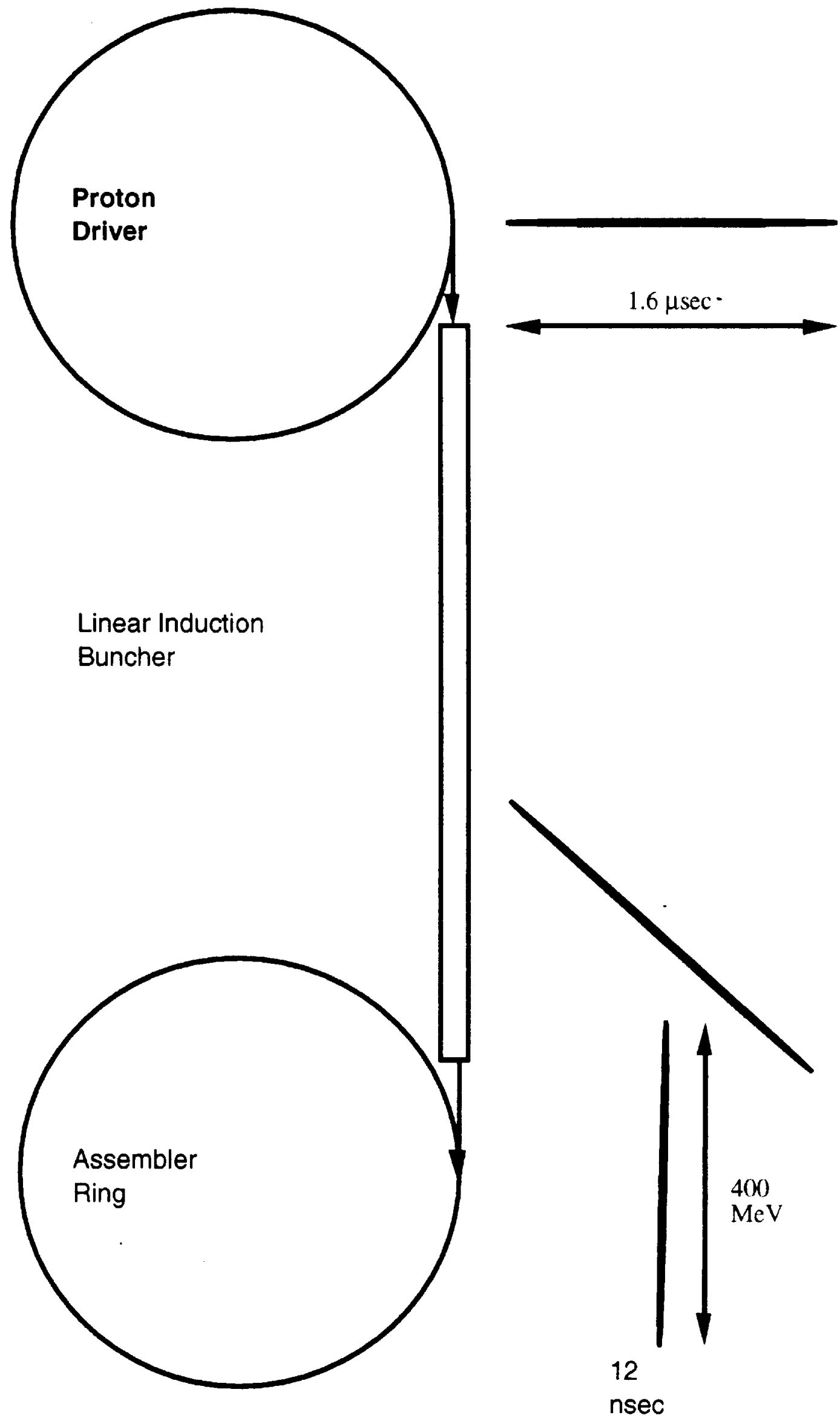

Figure 2. Assembler ring with linear bunching system and proton synchrotron. 


\section{REFERENCES}

[1] Private communication, Alfred W. Maschke, 1972. The tune of shift of several was demonstrated at injection in the AGS using the RF to bunch the beam transiently.

[2] K. Harkay"A Study of Longitudinal Instabilities and Emittance Growth in the Fermilab Booster Synchrotron", Perdue Univ. Thesis, Dec. 1993.

[3] L. J. Laslett, "On intensity limitations imposed by transverse space-charge effects in circular particle accelerators.", BNL Report BNL-7534, p.324 (1963).

[4] Grahame Rees, private communication, 1995.

[5] R. Baartman, in Proc. of the Workshop on Accelerators for Future Spallation Neutron Sources, Santa Fe, NM, 1993, LA-UR-93-1356.

[6] J.E.Griffin, "Aspects of Operation of the Fermilab Booster RF System at Very High Intensity", Fermilab TM-1968.

[7] M. Popovic, Private Commuication, September 1995.

[8] C. Johnstone, Private communication, September 1995.

[9] S.Y. Lee, K. Y. Ng, and D.Trbojevic, Phys. Rev. E 48 p3040, Ocotber 1993.

[10] Grahame Rees, ibid.

[11] R. J. Noble, "1 GeV Linac for Proton Driver of Muon Collider", Fermilab, June, 1995.

[12] J. Norem, Private Communication, October 1995. 